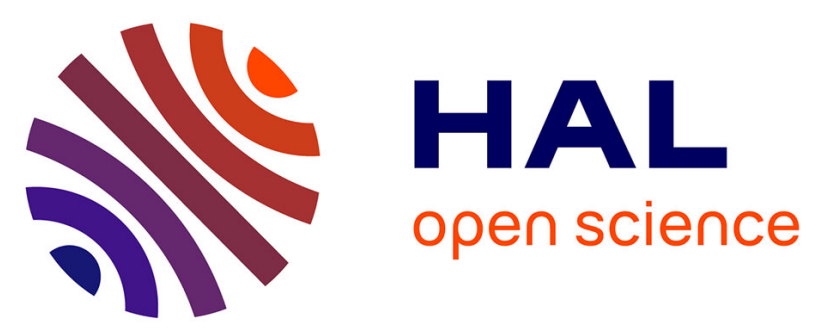

\title{
Interplay between collective pinning and artificial defects on domain wall propagation in $\mathrm{Co} / \mathrm{Pt}$ multilayers
}

G Rodríguez-Rodríguez, Jl Menéndez, A Hierro-Rodriguez, A Pérez-Junquera, N Montenegro, D Ravelosona, Jm Alameda, M Vélez

\section{- To cite this version:}

G Rodríguez-Rodríguez, Jl Menéndez, A Hierro-Rodriguez, A Pérez-Junquera, N Montenegro, et al. Interplay between collective pinning and artificial defects on domain wall propagation in $\mathrm{Co} / \mathrm{Pt}$ multilayers. Journal of Physics D: Applied Physics, 2010, 43 (30), pp.305002. 10.1088/0022$3727 / 43 / 30 / 305002$. hal-00569659

\section{HAL Id: hal-00569659 \\ https://hal.science/hal-00569659}

Submitted on 25 Feb 2011

HAL is a multi-disciplinary open access archive for the deposit and dissemination of scientific research documents, whether they are published or not. The documents may come from teaching and research institutions in France or abroad, or from public or private research centers.
L'archive ouverte pluridisciplinaire HAL, est destinée au dépôt et à la diffusion de documents scientifiques de niveau recherche, publiés ou non, émanant des établissements d'enseignement et de recherche français ou étrangers, des laboratoires publics ou privés. 


\title{
Interplay between collective pinning and artificial defects on domain wall propagation in Co/Pt multilayers
}

\author{
G Rodríguez-Rodríguez, ${ }^{1} \mathrm{JL}$ Menéndez, ${ }^{2}$ A Hierro-Rodriguez, ${ }^{1}$ A Pérez-

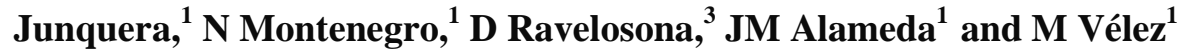 \\ ${ }^{1}$ Dept. Física, Universidad de Oviedo-CINN, 33007 Oviedo, SPAIN \\ 2 Centro de Investigación en Nanomateriales y Nanotecnología (CINN). \\ Principado de Asturias - Consejo Superior de Investigaciones Científicas \\ (CSIC) - Univ. Oviedo (UO). Parque Tecnológico de Asturias, 33428 Llanera, \\ Spain \\ ${ }^{3}$ Institut d'Electronique Fondamentale, UMR CNRS 8622, Univ. Paris Sud, \\ 91405 Orsay Cedex, FRANCE \\ E-mail: $\underline{\text { mvelez@uniovi.es }}$
}

\begin{abstract}
The interplay between collective pinning on intrinsic structural defects and artificial pinning at a patterned hole is studied in magnetic multilayers with perpendicular anisotropy. The pinning strength of a patterned hole is measured through its efficiency to stop domain wall propagation into a consecutive unpatterned nanowire section (using antisymmetric magnetoresistance to detect the direction of domain wall propagation) whereas collective pinning is characterized by the field dependence of domain wall velocity. Close to room temperature, collective pinning becomes weaker than artificial pinning so that pinning at the hole compensates nucleation-pad geometry, blocking domain wall propagation across the nanowire.
\end{abstract}

PACS: 75.60.Ch; 75.75.-c; 73.50.Jt 


\section{Introduction}

The study of domain wall (DW) propagation in magnetic nanostructures is a key issue for applications in logic/memory devices [1,2]. Depending on the application, fast DW propagation along large distances is needed in certain sections of the magnetic circuit as well as efficient and localized pinning centers with different types of pinning geometries [3-7]. In perpendicular anisotropy $\mathrm{Co} / \mathrm{Pt}$ multilayers the relevant mode for magnetization reversal is dominated by $\mathrm{DW}$ nucleation followed by rapid DW propagation [8]. At low fields, the competition between DW elastic energy and the disordered pinning potential induced by intrinsic quenched disorder in the multilayer results in a collective pinning regime for DW propagation [9]. Many structural induced factors are found to have a direct effect on DW velocities: for example, light ion irradiation reduces collective pinning $[10,11]$ resulting in faster DW propagation whereas slower DW motion can be obtained by enhancing the strength of collective pinning with strategies such as increasing the Co layer thickness [12], or fabricating arrays of Co dots on top of the multilayers [13]. However, the depinning fields resulting from artificial pinning centers are usually much smaller than those of intrinsic quenched disorder $[13,14]$ making it difficult to fabricate tailored pinning potentials for DW motion in these perpendicular anisotropy multilayers.

Ratchet effects induced by the asymmetry of the DW pinning potential [6] constitute a case of particular interest in the control of DW propagation for the fabrication of domain wall diodes [7] but, up to now they have been mostly studied in samples with in plane anisotropy. These experiments require the design of effective local pinning centers, a careful understanding of the different sources of asymmetry present in a magnetic circuit (nucleation pads, shape and location of pinning centers) and the development of detection techniques that determine not only DW speed but also its propagation direction. Kerr microscopy has been the preferred technique for samples with in plane magnetization [6,7] but, for perpendicular anisotropy multilayers, the recently discovered antisymmetric magnetoresistance can provide information on DW propagation sense in a much simpler way $[15,16]$. 
In this work, we have studied DW propagation in patterned $\mathrm{Co} / \mathrm{Pt}$ multilayers as a function of temperature and geometry in order to find a suitable regime in which the magnetic behaviour of the multilayer is dominated by a localized pinning potential: at low temperatures collective pinning by quenched disorder dominates the behaviour of the magnetic circuit but, as temperature increases, the strength of collective pinning in the multilayer decreases steeply so that a regime is reached in which a patterned hole can effectively block forward DW propagation, compensating nucleation pad asymmetry.

\section{Experimental}

$\mathrm{Co} / \mathrm{Pt}$ wires have been fabricated from high quality epitaxial $\mathrm{Pt}(3 \mathrm{~nm}) /[\mathrm{Co}(0.6 \mathrm{~nm}) / \mathrm{Pt}(1 \mathrm{~nm})]_{4} / \mathrm{Pt}(3 \mathrm{~nm})$ multilayers with perpendicular magnetic anisotropy in which magnetization reversal takes place by domain wall motion following rare nucleation events $[8,14]$. A combination of e-beam lithography and an etching process through $\mathrm{Ar}^{+}$milling
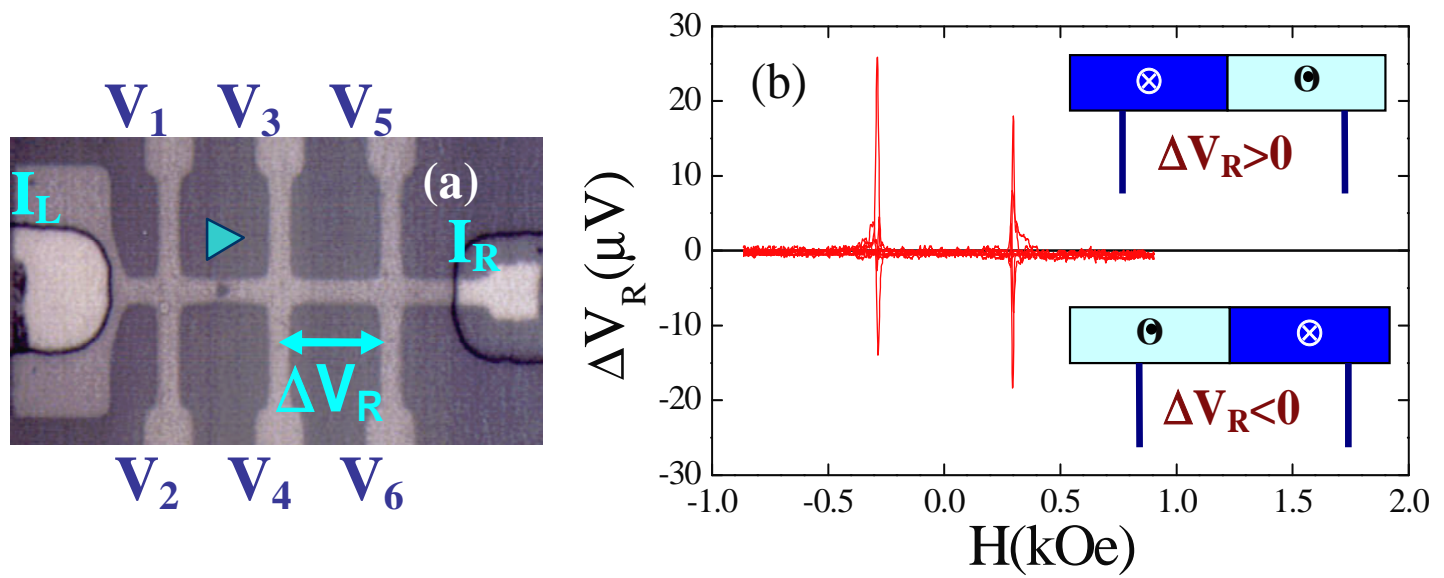

Figure 1. (colour online) (a) Micrograph of the $5 \mu \mathrm{m}$ wide patterned $\mathrm{Co} / \mathrm{Pt}$ wire indicating contact geometry for magnetoresistance measurements and the position of the triangular hole: $I_{L}$ and $I_{R}$ indicate the left/right nucleation pads, also used as current contacts; $\mathrm{V}_{1}$ to $\mathrm{V}_{6}$ indicate the contact pads used for voltage measurements; $\Delta \mathrm{V}_{\mathrm{R}}=\mathrm{V}_{6}-\mathrm{V}_{4}$ is the voltage signal used throughout this work; blue triangle indicates the position of the patterned hole in the wire. (b) Field dependence of magnetoresistance in a $5 \mu \mathrm{m}$ wide $\mathrm{Co} / \mathrm{Pt}$ wire at room temperature (the results of three different field loops are included). Sketches indicate magnetization configuration around DW for positive/negative magnetoresistance peaks. 
[16] has been used to fabricate a $40 \mu \mathrm{m}$ long wire of width $w=5 \mu \mathrm{m}$ (see Fig. 1a), together with 2 longitudinal contact pads for current injection (Labelled $I_{L}$ and $I_{R}$ in the figure) and three pairs of transverse contact pads spaced at $20 \mu \mathrm{m}$ intervals to allow for voltage measurements $\left(\mathrm{V}_{1}\right.$ to $\left.\mathrm{V}_{6}\right)$. In a second optical lithography step $\mathrm{Al}$ contacts are sputtered onto the $\mathrm{Co} / \mathrm{Pt}$ voltage/current pads in order to make electrical connections into the sample. The $\mathrm{Co} / \mathrm{Pt}$ contact pads, due to their larger area in comparison with the wire, are also of use as preferred nucleation sites for DWs in the reversal process of the system [14]. Two sources of asymmetry in the magnetic behavior are introduced in the system: first, the DW nucleation pads attached at both sides of the wire are unequal, with the largest at the left side (the ratio of small pad/large pad area is approximately 0.05 ), so that, in a nucleation dominated magnetization reversal process, the most likely sense of DW propagation will be from left to right (forward sense in the following); second, a large triangular hole of side $a=3 \mu \mathrm{m}$ is patterned on the left $\mathrm{Co} / \mathrm{Pt}$ wire section in order to stop forward DW propagation.

A continuous nanowire without the triangular hole has also been fabricated from the same $\mathrm{Co} / \mathrm{Pt}$ multilayer as a reference. A $613 \mathrm{~Hz} \mathrm{AC}$ current current is injected along the $\mathrm{Co} / \mathrm{Pt}$ wire and the voltage signal between the selected pair of contacts is measured with a lock-in detection technique [14]. In this work, all the data presented correspond to $\Delta \mathrm{V}_{\mathrm{R}}=\mathrm{V}_{6}-\mathrm{V}_{4}$, i.e. to domain wall propagation in the unpatterned right wire section. The magnetic field $\mathrm{H}$ is applied perpendicular to sample plane with a 4 kOe electromagnet.

Figure 1(b) shows several room temperature magnetoresistance hysteresis loops $\left(\Delta V_{R}\right.$ vs. $\mathrm{H}$ ), of the patterned $\mathrm{Co} / \mathrm{Pt}$ nanowire measured along the uninterrupted right wire section (see sketch in Fig. 1(a)). The curves present a series of narrow peaks around \pm 290 Oe (which is the sample coercivity at this temperature) but, different from typical anisotropic magnetoresistance, these peaks are either positive or negative in a random order. These features in the magnetoresistance peaks are the characteristic signature of antisymmetric magnetoresistance $[15,17]$ : in a geometric configuration of mutually perpendicular magnetization, current and DW (as is the case here), the electric fields related to extraordinary Hall effect at both sides of the 
wall give rise to an extra contribution at the magnetoresistance contacts, the sign of which depends on the sense of magnetization rotation around the DW, as sketched in Fig. 1(b). Therefore, for a given branch of the hysteresis loop, the positive/negative peaks observed in this figure can be attributed to different DW propagation events in which the wire magnetization is reversed by a DW propagating either in the forward or backward direction.

\section{Results and Discussion}

Time resolved magnetoresistance curves have been used to obtain information about the number of Forward/Backward DW propagation events, i.e. positive/negative magnetoresistance peaks (see inset of Fig.2). In order to ensure repeatability of the measurements, the sample is first saturated along one direction and, then, a constant magnetic field slightly below the coercivity one is applied in the opposite sense and the time resolved magnetoresistance signal in the uninterrupted right wire section is recorded. There are two clear advantages for this measurement configuration in comparison with characterization of DW propagation across the

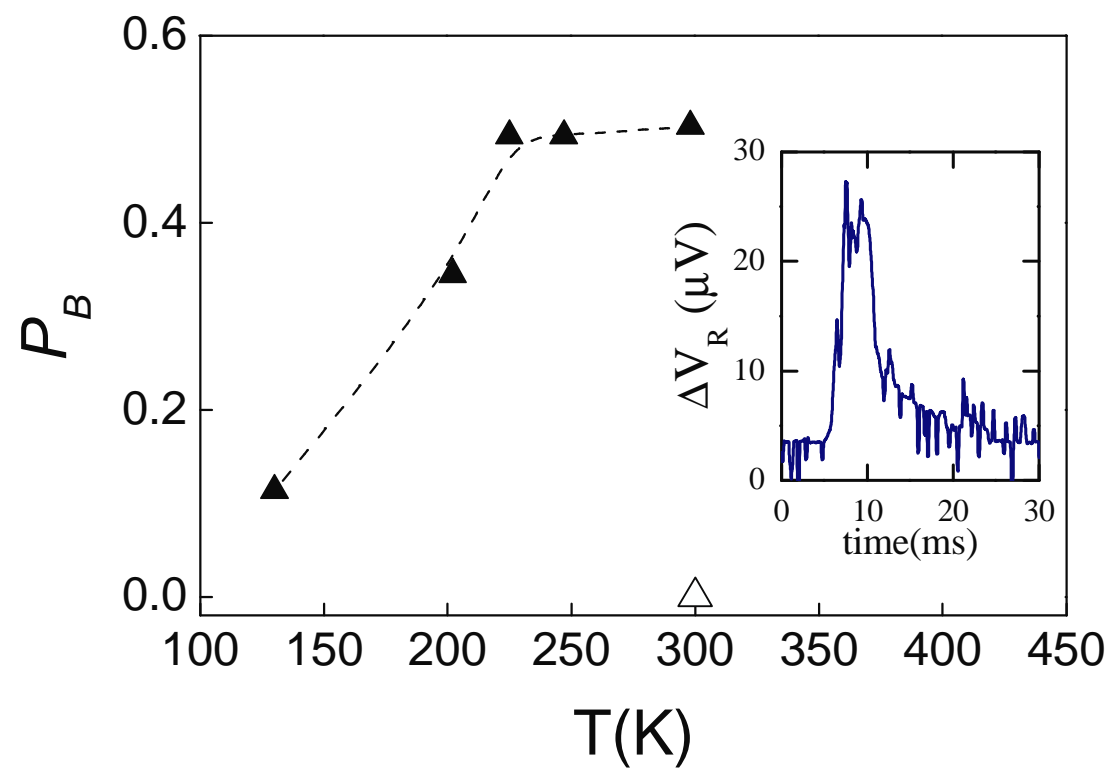

Figure 2. (colour online) Probability for backward domain wall propagation for a patterned and unpatterned wire (filled and hollow triangles, respectively) measured over a hundred propagation events at different constant temperatures. Dashed line is a guide to the eye. Inset is a time resolved magnetoresistance curve taken at $T=250 \mathrm{~K}$ and $H=300$ Oe showing a forward DW propagation event (positive magnetoresistance peak). 
left patterned wire segment: first, the sensitivity to the magnetoresistance peaks related to DW motion is much better since the presence of the hole increases the resistance of the patterned wire by an order of magnitude; second, the effects of collective and artificial pinning can be characterized by two independent experimental magnitudes: domain wall propagation times (given by the width of the time resolved magnetoresistance peaks) and DW propagation sense (given by the sign of these peaks). In this way, we can avoid the difficulty to define average DW velocities due to the stochastic nature of the DW propagation process [18-20] when different kind of pinning centers are present in the same nanowire section.

At low temperatures, most propagation events correspond to Forward DW motion, reflecting the different areas of the right and left nucleation pads but, as temperature increases, a larger number of negative magnetoresistance peaks (corresponding to backward DW propagation) are observed. On the contrary, only forward propagation events were detected in a similar experiment with a Co/Pt wire of identical geometry but without the patterned hole [16] as expected from nucleation pad geometry. Thus, the probability of backward DW propagation can be taken as a measure of the pinning efficiency of the patterned hole. The probability of Backward DW motion $P_{B}$ may be defined as

$$
P_{B}=\mathrm{N}^{-} /\left(\mathrm{N}^{-}+\mathrm{N}^{+}\right)
$$

Where $\mathrm{N}^{-}$and $\mathrm{N}^{+}$are the number of negative/positive magnetoresistance peaks, respectively, and $\mathrm{N}_{\mathrm{T}}=\mathrm{N}^{-}+\mathrm{N}^{+}$is the total number of events observed (over a hundred at each temperature). With this definition the probability of forward propagation $P_{F}$ is simply given by $P_{F}=1-P_{B}$. The temperature evolution of $P_{B}$ is shown in Figure 2: At $T=130 \mathrm{~K}, P_{B}=0.11$ so that the ratio of backward/forward probability is $P_{B} / P_{F}=0.12$ (close to the areal ratio of left/right nucleation pads 0.05 ) whereas for temperatures above $225 \mathrm{~K} P_{B}$ becomes essentially equal to 0.5 within the experimental accuracy (i.e. forward and backward propagation events are observed randomly with equal probability). Thus, at low temperatures the hole pinning efficiency is found to be very low but it becomes increasingly important as $T$ increases. This value of $P_{B} \approx 0.5$ may be understood taking into account the possibility of DW nucleation also at the lateral voltage pads 
as follows: if DW propagation across the hole is completely interrupted, the observed DW propagation events may come either from DW nucleation at $\mathrm{V}_{3} / \mathrm{V}_{4}$ (forward propagation) or from DW nucleation at $\mathrm{V}_{5} / \mathrm{V}_{6} / \mathrm{I}_{\mathrm{R}}$ (backward propagation); then, taking into account the different contact pads areas, the probabilities of forward/backward propagation should be almost the same. Thus the results presented in Fig. 2 point to an effective blocking of forward DW propagation across the patterned bridge section by the triangular hole for temperatures above $225 \mathrm{~K}$.

In order to understand this behavior, the temperature dependence of collective pinning from quenched disorder in the $\mathrm{Co} / \mathrm{Pt}$ multilayer must be considered. This has been characterized in the usual way by the field dependence of DW velocities $v(H)[9,14]$, derived from DW propagation times. Figure 3(a) shows several $v(H)$ curves measured at different temperatures in the range $100 \mathrm{~K}$ to $300 \mathrm{~K}$. The typical collective pinning behavior of a $1 \mathrm{D}$ wall propagating in a 2D disordered medium [21] is given by the creep law

$$
v(H)=v_{0} \exp \left[-\beta U_{0}\left(H_{0} / H\right)^{1 / 4}\right]
$$

where $U_{0}$ and $H_{0}$ are, respectively, the characteristic pinning energy and the critical field, first
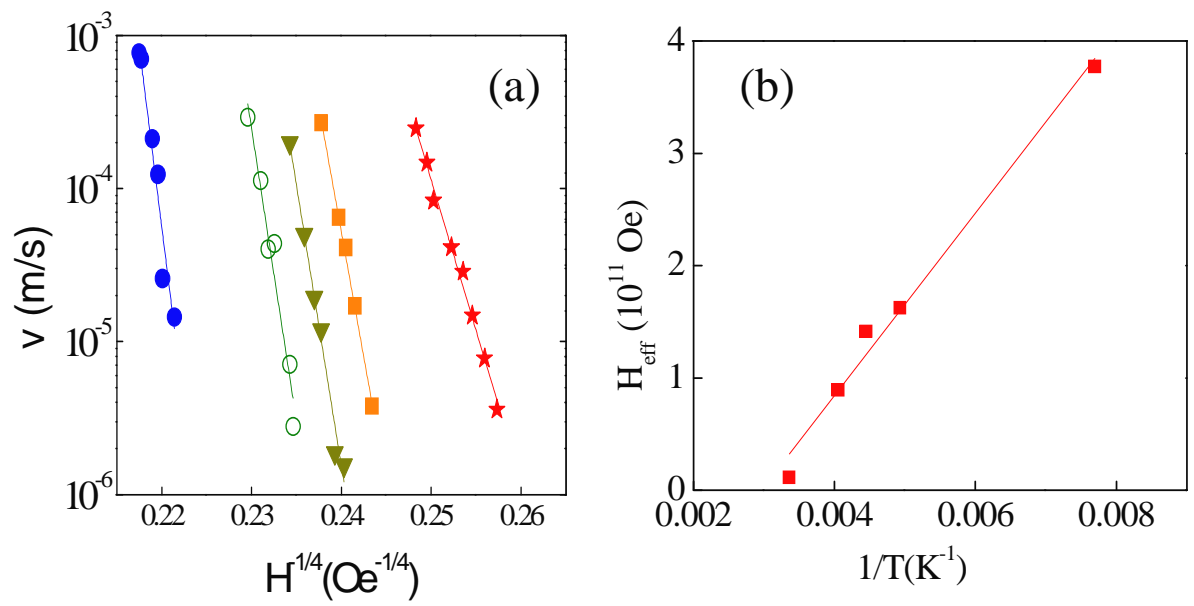

Figure 3. (colour online) a) Semilogarithmic plot of DW velocity vs. $H^{1 / 4}$ showing the typical creep behavior of a 1D line in a 2D weakly disordered medium: circles, $T=130 \mathrm{~K}$; hollow circles, $T=200 \mathrm{~K}$; triangles down, $T=220 \mathrm{~K}$; squares, $T=250 \mathrm{~K}$; stars, $T=300 \mathrm{~K}$. (b) Temperature dependence of effective creep field for domain wall propagation. Solid line is a linear fit to $H_{\text {eff }} \propto$ $(1 / T-1 / 330 \mathrm{~K})$. 
reported in [9] in the context of DW motion. Thus, the linear behaviour of $\log v v s H^{1 / 4}$ observed in Fig. 3 is a clear indication that DW motion in the unpatterned bridge occurs by a creep process over a random pinning potential. For intrinsic quenched disorder, the critical field is given by $H_{0}{ }^{\mathrm{int}}=\sigma / M_{\mathrm{s}}\left(\xi / \mathrm{L}_{\mathrm{c}}{ }^{2}\right)$ with $\sigma$ the wall energy, $M_{\mathrm{s}}$ the saturation magnetization, $\xi$ the quenched disorder correlation length and $L_{\mathrm{c}}$ the Larkin length related with the size of the rigid microscopic wall segments that interact with disorder. The effective field $H_{\text {eff }}=\left(\beta U_{0}\right)^{4} H_{0}$, calculated from the slopes of the $v(H)$ curves (see Fig.3(b)), shows a strong temperature dependence, decreasing an order of magnitude from $H_{\text {eff }}=3.8 \times 10^{11}$ Oe at $T=130 \mathrm{~K}$ to $H_{\text {eff }}=$ $10^{10} \mathrm{Oe}$ at room temperature (no effect of the patterned hole is observed in $H_{\text {eff }}$, as expected, since the observed DW propagation events occur in the continuous right nanowire section as indicated in Fig. 1a).

$H_{\text {eff }}(T)$ follows roughly a $\left(1 / T^{-}{ }^{1} / 330 \mathrm{~K}\right)$ law, as indicated by the linear fit in Fig. 3(b). This is in contrast with the results in simple $\mathrm{Pt} / \mathrm{Co} / \mathrm{Pt}$ trilayers $\left(\mathrm{t}_{\mathrm{Co}}=0.5-1 \mathrm{~nm}\right)[10,14]$ in which $H_{\mathrm{eff}}$ takes much lower values, $H_{\mathrm{eff}} \approx 10^{6}-10^{8} \mathrm{Oe}$, and is only weakly temperature dependent. The larger values of $H_{\text {eff }}$ could be attributed to a larger effective thickness in the multilayers since $H_{\text {eff }}$ is strongly dependent on Co thickness [12]. Thus, the observed decrease in $H_{\text {eff }}(T)$ could be taken as a measure of the temperature dependence of interlayer coupling that determines the number of Co layers in the multilayer that reverse simultaneously. It is interesting to mention that the multilayer coercivity decreases monotonically from 450 Oe at $120 \mathrm{~K}$ to 290 Oe at room temperature, which is a further signature of a reduction in coupling strength as a function of temperature [22]. Similar transitions in the reversal mode from full multilayer reversal to layer by layer reversal have also been qualitatively observed in $\mathrm{Co} / \mathrm{Pt}$ multilayers with thicker $\mathrm{Pt}$ layers but in a lower temperature range [23].

The comparison between the results of Figs. 2 and 3 shows that as the strength of collective pinning is reduced close to room temperature, the patterned hole becomes more effective in blocking domain wall propagation. The link between these two effects may be understood as follows: Previous works in patterned nanowires with large artificial edge roughness [14] have 
shown that, at this scale, pinning occurs via a topography field $H^{\text {top }}$, related to the changes in DW length upon traversing the defect that adds to the intrinsic critical field of the wire. Then, in the unpatterned right wire section, $H_{0}^{\text {right }}=H_{0}{ }^{\text {int }}$ whereas in the left patterned wire section $H_{0}^{\text {left }}=$ $H_{0}^{\text {int }}+H^{\text {top }}$. The topography field is related to the hole geometry and, for the simple geometry of a triangular hole, should be of the order $H^{\text {top }} \approx \sigma / M_{\mathrm{s}} 1 /(w-a) . H_{0}{ }^{\mathrm{int}}$ is usually much larger than $H^{\text {top }}$ even for large patterned defects [14], but should present stronger temperature dependence than $H^{\mathrm{top}}$ since $L_{\mathrm{c}}$ diverges as the strength of intrinsic pinning is reduced [9]. Thus, as $H_{0}{ }^{\mathrm{int}}$ decreases, the contribution of $H^{\text {top }}$ becomes significant enough to induce a reduction in DW velocity in the patterned wire section that compensates the larger nucleation probability due to pad geometry, leading to the observed changes in the balance of forward/backward propagation events.

\section{Conclusion}

In summary, the pinning efficiency of a patterned hole has been evaluated using the applied criteria given by the probability to find DW motion in an unpatterned wire section after the defect. It is found that the interplay between collective pinning from quenched disorder and artificial induced pinning by a large patterned hole determines the temperature range in which the magnetic behaviour of the system is determined by the artificial defect.

\section{Acknowledgements}

Work supported by Spanish MICINN (FIS2008-06249), by Principado de Asturias FICYT (PCTI IB08-106) and by European Community (MERG-CT-2004-513625). 


\section{References}

[1] Allwood DA, Xiong G, Faulkner CC, Atkinson D, Petit D and Cowburn RP 2005 Science 3091688

[2] Parkin SSP, Hayashi M and Thomas L 2008 Science 320190

[3] Thomas L, Rettner C, Hayashi M, Samant MG, Parkin SSP, Doran A and Scholl A 2005 Appl. Phys. Lett. 87262501

[4] Himeno A, Kasai S and Ono T 2006 J. Appl. Phys 99 08G304

[5] Martinez E, Lopez-Diaz L, Alejos O and Torres L 2009 J. Appl. Phys. 106043914

[6] Pérez-Junquera A, Marconi VI, Kolton AB, Alvarez-Prado LM, Souche Y, Alija A, Velez M, Anguita JV, Alameda JM, Martin JI, and Parrondo JMR 2008 Phys. Rev. Lett. 100 037203

[7] Allwood DA, Xiong G, and Cowburn RP 2004 Appl. Phys. Lett. 852848

Ilgaz D, Klaui M, Heyne L, Zisner F, Krzyk S, Fonin M, Rudiger U, Backes D and Heyderman L 2008 Appl. Phys. Lett. 93132503

[8] Pommier J, Meyer P, Pénissard G, Ferré J, Bruno P and Renard D 1990 Phys. Rev. Lett. 65 2054

[9] Lemerle S, Ferré J, Chappert C, Mathet V, Giamarchi T, and Le Doussal P 1998 Phys. Rev. Lett. 80849

[10] Cayssol F, Menéndez JL, Ravelosona D, Chappert C, Jamet JP, Ferré J, and Bernas H 2005 Appl. Phys. Lett. 86022503

[11] San Emeterio Alvarez L, Burnell G, Marrows CH, Wang KY, Blackburn AM, and Williams DA 2007 J. Appl. Phys. 101 09F508

[12] Metaxas PJ, Jamet JP, Mougin A, Cormier M, Ferré J, Baltz V, Rodmacq B, Dieny B, and Stamps RL 2007 Phys. Rev. Lett. 99217208

[13] Metaxas PJ, Zermatten PJ, Jamet JP, Ferré J, Gaudin G, Rodmacq B, Schuhl A, and Stamps RL 2009 Appl. Phys. Lett. 94132504 
[14] Cayssol F, Ravelosona D, Chappert C, Ferré J, and Jamet JP 2004 Phys. Rev. Lett. 92 107202

[15] Cheng XM, Urazhdin S, Tchernyshyov O, Chien CL, Nikitenko VI, Shapiro AJ, and Shull RD 2005 Phys. Rev. Lett. 94017203

[16] Rodriguez-Rodriguez G, Menéndez JL, Pérez-Junquera A, Hierro-Rodriguez A, Montenegro N, Ravelosona D, Alameda JM and Vélez M 2010 J. Phys.: Conf. Ser. 200, 042021

[17] Xiang G and Samarth N 2007 Phys. Rev. B 76054440

[18] Burrowes C, Mihai AP, Ravelosona D, Kim JV, Chappert C, Vila L, Marty A, Samson Y, García-Sanchez F, Buda-Prejbeanu LD, Tudosa I, Fullerton EE and Attané JP 2010 Nature Physics 617

[19] Im M, Blocklage L, Fischer P and Meier G 2009 Phys. Rev. Lett. 102147204

[20] Burrowes C, Ravelosona D, Chappert C, Mangin S, Fullerton EE, Katine JA, and Terris BD 2008 Appl. Phys. Lett. 93172513

[21] Müller M, Gorokhov DA, and Blatter G 2001 Phys. Rev. B 63184305

[22] Knepper W and Yang FY 2005 Phys. Rev. B 71224403

[23] Xie XP, Zhao XW, Knepper JW, Yang FY, and Sooryakumar R 2007 Phys. Rev. B 76 184433 